\title{
Evaluation of nine 3D printing materials as tissue equivalent materials in terms of mass attenuation coefficient and mass density
}

\author{
Moayyad Alssabbagh ${ }^{1, *}$, Abd Aziz Tajuddin ${ }^{1,2}$, Mahayuddin bin Abdul Manap ${ }^{1}$, Rafidah Zainon ${ }^{1}$ \\ ${ }^{1}$ Advanced Medical and Dental Institute, Universiti Sains Malaysia, Pulau Pinang, Malaysia \\ ${ }^{2}$ School of Physics, Universiti Sains Malaysia, Pulau Pinang, Malaysia
}

\section{A R T I C L E I N F O}

\section{Article history:}

Received 8 February 2017

Received in revised form

2 August 2017

Accepted 4 August 2017

\section{Keywords:}

Attenuation values

Mass density

elemental composition

ICRU

Phantom

\begin{abstract}
A B S T R A C T
The main objective of this study is to evaluate the mass attenuation coefficients of nine 3D printing materials and to verify the best 3D printing materials to simulate the human soft tissue. The elementary compositions of nine 3D printing materials were evaluated using SEM-EDS machine. These 3D printing materials are Polylactic Acid (PLA), Acrylonitrile Butadiene Styrene (ABS), Polycarbonate (PC), Polyethylene terephthalate (PETG), Thermoplastic elastomers (TPE), Thermoplastic Polyurethane (TPU), High Impact Polystyrene (HIPS), Polyamide-Nylon (PA) and Wood. The mass attenuation coefficient of each 3D printing material was calculated by inserting its elemental composition into the XCom database, which provided and supported by the National Institute of Standards and Technology (NIST). The x-ray attenuation properties of nine different human organs tissue (brain, breast, eye lens, heart, kidney, liver, skin, testis and thyroid) was analyzed using the values listed in the International Commission on Radiation Units and Measurements - ICRU, report 44 .The percentage difference between the mass density of each material and each organ tissue was evaluated. The results were compared to find the best material that could mimic the human soft tissue organs in terms of the attenuation values and density. These results indicate that the 3D wood material can be used to simulate the brain, breast, testis, kidney, thyroid, and the TPU material can be used to mimic eye lens, heart, liver and skin in terms of the total mass attenuation coefficient and mass density. This study reveals that the 3D printing materials can be used to construct human phantoms whereas they are commercially available and cost effective material compared to current commercial tissue equivalent materials.
\end{abstract}

(C) 2017 The Authors. Published by IASE. This is an open access article under the CC BY-NC-ND license (http://creativecommons.org/licenses/by-nc-nd/4.0/).

\section{Introduction}

Phantoms actually used to impersonate the human tissues. The phantom constructed from materials called tissue equivalent materials which the properties of these materials alter with the energy of the radiation interacting with them. Therefore, the materials of a phantom for use in radiotherapy could be different than a phantom designed for diagnostic radiology (DeWerd and Kissick, 2014).

The most famous material of phantoms that still used until these days is water due to its properties to simulate the human tissue. However, it has very

\footnotetext{
* Corresponding Author.

Email Address: sabmomo75@gmail.com (M. Alssabbagh) https://doi.org/10.21833/ijaas.2017.09.024

2313-626X/C) 2017 The Authors. Published by IASE.

This is an open access article under the CC BY-NC-ND license

(http://creativecommons.org/licenses/by-nc-nd/4.0/)
}

basic geometry shape, such as water tank and cylinders. Wax can be replaced for water but it has different types with different formulations that affect the reading consistency. For low radiation energy, wax shows different tissue properties. This solved by adding materials with high atomic numbers to the mixture, but still has uncertainty degree of tissue equivalency. Wood has been used during the late 1930s until today to simulate the soft tissue, but it has mostly the same problems of wax material (Shakhreet et al., 2009; Tousi et al., 2014).

The Polymethylmethacrylate (PMMA) is the most prominent material used nowadays as a tissue equivalent material due to its homogeneity and similarity of the human tissue (Ferreira et al., 2010; Borcia and Mihailescu, 2008).

Other phantoms made of real human tissue, such as the Temex ${ }^{\mathrm{TM}}$ phantom and the Rando ${ }^{\circledR}$ phantom developed by Stacey et al. (1961) and Alderson et al. (1962). Both phantoms contain real human skeleton 
planted in equivalent tissue material. Now days, The Phantoms have evolved with time and new materials are used, such as polyurethanes and epoxy resins, which provide a higher reproducibility and quality. This improvement allows using the phantoms over a different energies range.

Choosing the material of a phantom is a very sensitive and critical stage. The phantom material that is chosen should have a reasonable accuracy to represent the physical and radiological properties of the selected tissue.

The radiation interaction with the material varies according to its mass density, atomic number and the type of incident radiation, such as photons and charged particles. The mechanisms of absorption or attenuation inside the material can give different results at different energies range, particularly in diagnostic and therapeutic range. This is because of different events (photoelectric effect, Compton scattering, and pair production) that could happen during the interaction with the matter.

There are several factors need to be taken into account when choosing the phantom materials, such as the mass density, the effective atomic number, the electron density of the materials and the most physical property that widely used to investigate the equivalence of tissue is the mass attenuation coefficient $(\mu / \rho)$. This parameter indicates how much energy is penetrated over a volume of a material (DeWerd and Kissick, 2014).

The linear attenuation coefficient $\mu$ considered as the most useful variable to describe the relation between the penetration of radiation and the absorber material along its path per unit length inside the matter. This is strongly, depends on the atomic number of the material and the energy of the incident radiation. Dividing the linear attenuation coefficient $\mu$ by the density of the absorber $\rho$ is defined as mass attenuation coefficient, which measured by $\mathrm{m} 2 / \mathrm{kg}$ (Podgorsak, 2010; Beutel et al., 2000). Based on Hubbell (2006) theory the total mass attenuation coefficient $(\mu / \rho)$ as a combination of cross sections of the individual effect values for Rayleigh $\left(\sigma_{R}\right)$, photo-effect $\left(\sigma_{\tau}\right)$, pair production $(\kappa)$ including triplet production and Compton scattering $\left(\sigma_{c}\right)$ divided by the atomic mass unit $\left(m_{u}\right)$ and the relative atomic mass of the absorber $(A)$ (Hubbell, 2006) (Eq. 1).

$\frac{\mu}{\rho}=\frac{\sigma_{R}+\sigma_{\tau}+K+\sigma_{C}}{m_{u} A}$

Elementary composition and mass attenuation coefficient was used in many studies of new kinds of tissue substitute materials for simulating different human tissues (Akça and Erzeneoğlu, 2014; Jones et al., 2003; Ferreira et al., 2010; DeWerd and Kissick, 2014). It also has been used in the shielding building materials (glass, concrete, marble, fly ash, cement, lime, etc.) to investigate the interaction in such materials with radiation (Singh et al., 2004; Chanthima et al., 2012). To select the suitable equivalent tissue material, the results of elemental composition and mass attenuation coefficient usually compared with the values accomplished in the ICRU no. 44 (ICRU, 1989), which provides details of the elemental compositions, mass densities, electron densities and interaction data of all human body tissue. The most trusted software in mass attenuation coefficient calculation for many researchers nowadays is the XCOM photon crosssection database (Ferreira et al., 2010; Shakhreet et al., 2009; Tousi et al., 2014; Gerward et al., 2004; Akça and Erzeneoğlu, 2014; Singh et al., 2004; Alsabbagh et al., 2016), which provided and supported by the National Institute of Standards and Technology (NIST) based on Hubbell and Seltzer tables (Hubbell and Seltzer, 1996). The main objective of this study is to verify the best material of nine 3D materials used in 3D printing technology to simulate the human soft tissues.

\section{Materials and methods}

Two steps followed in this study. The first step is studying the attenuation properties and the mass density of nine various organs in the human body based on the publication of International Commission on Radiation Units and Measurements ICRU, report n 44. These organs were: brain, breast, eye lens, heart, kidney, liver, skin, testis and thyroid.

The second step is analyzing the attenuation properties of nine different 3D printing materials, which are commercially available and widely used in different 3D printing fields based on their elemental compositions, mass densities and mass attenuation coefficients. The materials analyzed in this study were Polylactic Acid (PLA), Acrylonitrile Butadiene Styrene (ABS), and Polycarbonate (PC), Polyethylene terephthalate (PETG), Thermoplastic elastomers (TPE), Thermoplastic Polyurethane (TPU), High Impact Polystyrene (HIPS), Polyamide-Nylon (PA) and Wood. The elemental compositions were obtained to calculate the total mass attenuation coefficient for each material. To get the mass fraction of each element of the nine selected materials, the scanning electron microscopy utilized in Energy Dispersive X-ray Spectroscopy (SEM-EDS) machine (Quanta FEG 650; FEI, Hillsboro, OR, USA) (Fig. 1) was used.

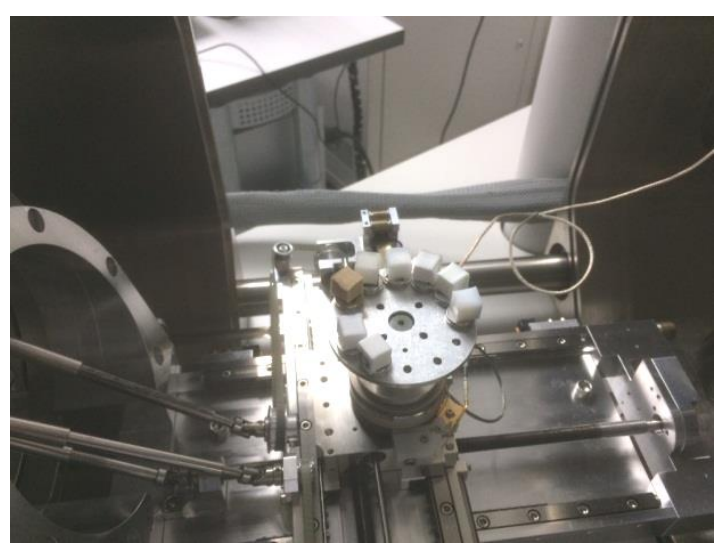

Fig. 1: The 3D materials under test to determine their elementary composition using SEM-EDS 
The results were then inserted into the online XCOM photon cross-section database software (Berger and Hubbell, 1999) to calculate the total mass attenuation coefficient of each 3D material at energies fall in the range of the diagnostic radiology field (10 keV to $150 \mathrm{keV}$ ).

The results were then compared with the values established in ICRU report no. 44 for each human organ tissue to determine the best tissue equivalent material for this energy interval.

\section{Results and discussion}

\subsection{Comparison of mass density}

Densities of each material under test, which provided by the manufacturer were compared with the densities of nine human organs that obtained from ICRU report no. 44. The percentage differences of each material in relation to ICRU's human organs were shown in Table 1 . The maximum absolute values of the percentage difference for brain, breast, eye lens, heart, kidney, liver, skin, testis and thyroid were $22.12 \%, 24.51 \%, 18.69 \%, 19.81 \%, 20.95 \%$, $19.81 \%, 16.51 \%, 22.12 \%$ and $20.95 \%$ respectively. The smaller percentage difference values for brain and testis was for the High Impact Polystyrene (HIPS), $0.48 \%$, Breast for wood (-1.96\%), Skin for Thermoplastic Polyurethane (TPU), $0.92 \%$ and eye lens, heart, kidney, liver and thyroid for Acrylonitrile Butadiene Styrene (ABS), -1.87\%, -0.95\%, $0 \%$, $0.945 \%$ and $0 \%$ respectively. In general, the results show quite good congruence of mass densities of the 3D materials with the mass densities of selected human organs.

Table 1: The percentage difference between the Mass densities of ICRU's human organs and the 3D materials

\begin{tabular}{|c|c|c|c|c|c|c|c|c|c|c|}
\hline & Organ & Brain & Breast & Eye lens & Heart & Kidney & Liver & Skin & Testis & Thyroid \\
\hline \multirow{2}{*}{ Material } & \multirow{2}{*}{ Density $\left(\mathrm{g} / \mathrm{cm}^{3}\right)$} & 1.04 & 1.02 & 1.07 & 1.06 & 1.05 & 1.06 & 1.09 & 1.04 & 1.05 \\
\hline & & \multicolumn{9}{|c|}{ Percentage difference (\%) (3D material/ICRU's human organ) } \\
\hline PLA & 1.25 & 20.19 & 22.55 & 16.82 & 17.92 & 19.05 & 17.92 & 14.68 & 20.19 & 19.05 \\
\hline ABS & 1.05 & 0.96 & 2.94 & -1.87 & -0.94 & 0.00 & -0.94 & -3.67 & 0.96 & 0.00 \\
\hline PC & 1.21 & 16.35 & 18.63 & 13.08 & 14.15 & 15.24 & 14.15 & 11.01 & 16.35 & 15.24 \\
\hline PETG & 1.27 & 22.12 & 24.51 & 18.69 & 19.81 & 20.95 & 19.81 & 16.51 & 22.12 & 20.95 \\
\hline TPE & 0.935 & -10.10 & -8.33 & -12.62 & -11.79 & -10.95 & -11.79 & -14.22 & -10.10 & -10.95 \\
\hline TPU & 1.1 & 5.77 & 7.84 & 2.80 & 3.77 & 4.76 & 3.77 & 0.92 & 5.77 & 4.76 \\
\hline HIPS & 1.045 & 0.48 & 2.45 & -2.34 & -1.42 & -0.48 & -1.42 & -4.13 & 0.48 & -0.48 \\
\hline PA - Nylon & 1.16 & 11.54 & 13.73 & 8.41 & 9.43 & 10.48 & 9.43 & 6.42 & 11.54 & 10.48 \\
\hline Wood & 1 & -3.85 & -1.96 & -6.54 & -5.66 & -4.76 & -5.66 & -8.26 & -3.85 & -4.76 \\
\hline
\end{tabular}

\subsection{Elemental composition and Total mass attenuation coefficient}

EMS-EDS machine was used to obtain the elemental composition of all selected materials as shown in Table 2 . Table 3 shows the elemental composition of the selected human organ as established in ICRU report no. 44. By inserting the weight fraction of each material and human organ into the web version of XCom software, the total mass attenuation coefficient was calculated for the energy range of $70 \mathrm{kVp}$ to $200 \mathrm{kVp}(30 \mathrm{keV}-150$ $\mathrm{keV}$ ), which most of medical diagnostic radiology practices fall in this energy interval. The total mass attenuation coefficient proportionally increases with the number of interactions of $x$-ray photons with the materials for this energy range. The ratios of the total mass attenuation coefficients between both of the 3D materials and the ICRU's human tissues were calculated, and the mean and the standard deviation of the ratios were obtained as shown in Table 4.

The results show the material that simulates each human organ, which indicated by the mean value closer to 1 . It can be seen from the results that all materials have good agreements with the human organs since all of them considered as a soft tissue. Thus, the Polycarbonate (PC) and the Polylacticacid (PLA) show a good agreement with all human organs under test in terms of the total mass attenuation coefficient.

Although the mass density suggested different materials as a substitute material of the selected human tissue as described in previous section, they didn't show a good agreement in the total mass attenuation coefficient, which considered as the most important quantity when it comes to the phantom materials that could be used in dosimetry (ICRU, 1989).

Table 2: The elemental compositions of the substitute materials

\begin{tabular}{cccccccccccccc}
\hline \multirow{2}{*}{ Material } & \multicolumn{10}{c}{ Elemental composition (percentage by mass) } \\
\cline { 2 - 14 } & $\mathrm{C}$ & $\mathrm{N}$ & $\mathrm{O}$ & $\mathrm{Na}$ & $\mathrm{Mg}$ & $\mathrm{Al}$ & $\mathrm{Si}$ & $\mathrm{S}$ & $\mathrm{Cl}$ & $\mathrm{K}$ & $\mathrm{Ca}$ & $\mathrm{Ti}$ \\
\hline TPE & 96.71 & - & 2.69 & 0.13 & - & 0.11 & - & 0.13 & 0.09 & - & 0.13 & - \\
Wood & 61.48 & - & 37.65 & - & 0.26 & - & 0.47 & - & 0.15 & - & - & - \\
PA & 69.53 & 13.16 & 17.31 & - & - & - & - & - & - & - & - & - \\
APS & 92.44 & - & 5.68 & 1.79 & - & - & - & 0.09 & - & - & - & - \\
HIPS & 97.88 & - & 1.88 & 0.11 & - & - & - & - & 0.13 & - & - & - \\
PLA & 54.76 & - & 44.99 & - & - & - & - & 0.13 & - & 0.12 & - & - \\
TPU & 66.63 & 8.98 & 24.13 & - & - & 0.06 & 0.10 & - & - & - & 0.09 & - \\
PC & 76.28 & - & 22.79 & - & - & - & - & 0.11 & - & - & - & 0.82 \\
PETG & 70.45 & - & 29.33 & 0.07 & - & - & - & 0.15 & - & - & - & - \\
\hline
\end{tabular}


Table 3: The listed elemental compositions in ICRU report no. 44 for each human organ under test

\begin{tabular}{ccccccccccccccc}
\multicolumn{11}{c}{ Elemental composition (percentage by mass) } \\
\hline Tissue & $\mathrm{H}$ & $\mathrm{C}$ & $\mathrm{N}$ & 0 & $\mathrm{Na}$ & $\mathrm{Mg}$ & $\mathrm{P}$ & $\mathrm{S}$ & $\mathrm{Cl}$ & $\mathrm{K}$ & $\mathrm{Ca}$ & $\mathrm{I}$ & $\mathrm{Fe}$ \\
Brain & 10.7 & 14.5 & 2.2 & 71.2 & 0.2 & - & 0.4 & 0.2 & 0.3 & 0.3 & - & - & - \\
Breast & 10.6 & 33.2 & 3 & 52.7 & 0.1 & - & 0.1 & 0.2 & 0.1 & - & - & - & - \\
Eye lens & 9.6 & 19.5 & 5.7 & 64.6 & 0.1 & - & 0.1 & 0.3 & 0.1 & - & - & - & - \\
Heart & 10.3 & 12.1 & 3.2 & 73.4 & 0.1 & - & 0.1 & 0.2 & 0.3 & 0.2 & - & - & 0.1 \\
Kidney & 10.3 & 13.2 & 3 & 72.4 & 0.2 & - & 0.2 & 0.2 & 0.2 & 0.2 & 0.1 & - & - \\
Liver & 10.2 & 13.9 & 3 & 71.6 & 0.2 & - & 0.3 & 0.3 & 0.2 & 0.3 & - & - & - \\
Skin & 10 & 20.4 & 4.2 & 64.5 & 0.2 & - & 0.1 & 0.2 & $03 \mathrm{C}$ & 0.1 & - & - & - \\
Testis & 10.6 & 9.9 & 2 & 76.6 & 0.2 & - & 0.1 & 0.2 & 0.2 & - & - & - & - \\
Thyroid & 10.4 & 11.9 & 2.4 & 74.5 & 0.2 & - & 0.1 & 0.1 & 0.2 & 0.1 & - & 0.1 & - \\
\hline
\end{tabular}

Table 4: The mean and the standard deviation of the ratios

\begin{tabular}{|c|c|c|c|c|c|c|c|c|c|}
\hline & \multicolumn{9}{|c|}{ Mean of the Ratio (3D material/ICRU's human organ) } \\
\hline & Brain & Bre & Eye lens & Heart & Kidney & Liver & Skin & Testis & oid \\
\hline PETG & $0.866 \pm 0.05$ & $0.895 \pm 0.01$ & $0.889 \pm 0.03$ & $0.857 \pm 0.05$ & $0.870 \pm 0.04$ & $0.870 \pm 0.05$ & $0.850 \pm 0.07$ & $.873 \pm 0.04$ & 0.05 \\
\hline PC & $.889 \pm 0.02$ & $0.920 \pm 0.02$ & $0.913 \pm 0$ & $0.880 \pm 0.02$ & $0.894 \pm 0.02$ & $0.893 \pm 0.02$ & $0.872 \pm 0.05$ & $.896 \pm$ & \pm 0.03 \\
\hline Wood & $880 \pm 0.03$ & $0.910 \pm 0$ & $903 \pm 0.01$ & $870 \pm 0.03$ & 0.884 & $0.884 \pm$ & $0.863 \pm$ & 887 & .04 \\
\hline TPU & $0.867 \pm 0.04$ & $.895 \pm$ & \pm 0.03 & $0.857 \pm 0.05$ & 0.871 & $0.870 \pm$ & $0.851 \pm$ & 0.873 & 0.05 \\
\hline PLA & $.884 \pm 0.03$ & $.914 \pm 0.01$ & $0.907 \pm 0.01$ & $0.874 \pm 0.03$ & 0.888 & $0.887 \pm 0$ & $0.867 \pm 0$ & $0.890 \pm$ & 0.86 \\
\hline HIPS & $0.840 \pm 0.08$ & $0.867 \pm 0.05$ & $0.861 \pm 0.06$ & $0.831 \pm 0.08$ & 0.84 & $0.843 \pm 0$ & $0.825 \pm 0.1$ & $0.846 \pm$ & 0.82 \\
\hline ABS & $841 \pm 0.07$ & $0.868 \pm 0.05$ & $.862 \pm 0.06$ & $0.832 \pm 0.08$ & $0.845 \pm 0.07$ & $0.844 \pm 0.07$ & $0.826 \pm 0.1$ & $0.847 \pm 0.07$ & $0.827 \pm 0.08$ \\
\hline PA & $0.857 \pm 0.06$ & $0.886 \pm 0.02$ & $0.879 \pm 0.04$ & $0.848 \pm 0.06$ & $0.861 \pm 0.05$ & $0.861 \pm 0.06$ & $0.842 \pm 0.08$ & $0.864 \pm 0.05$ & $0.843 \pm 0.06$ \\
\hline TPE & $0.847 \pm 0.07$ & $0.874 \pm 0.04$ & $0.868 \pm 0.05$ & $0.838 \pm 0.07$ & $0.851 \pm 0.07$ & $0.850 \pm 0.07$ & $0.832 \pm 0.09$ & $0.853 \pm 0.06$ & $0.832 \pm 0.07$ \\
\hline
\end{tabular}

According to the better values of the percentage differences between the densities of the materials and the human organs under test, Fig. 2 illustrates the mass attenuation values of (a) HIPS material with brain and testis, (b) wood with Breast, (c) TPU

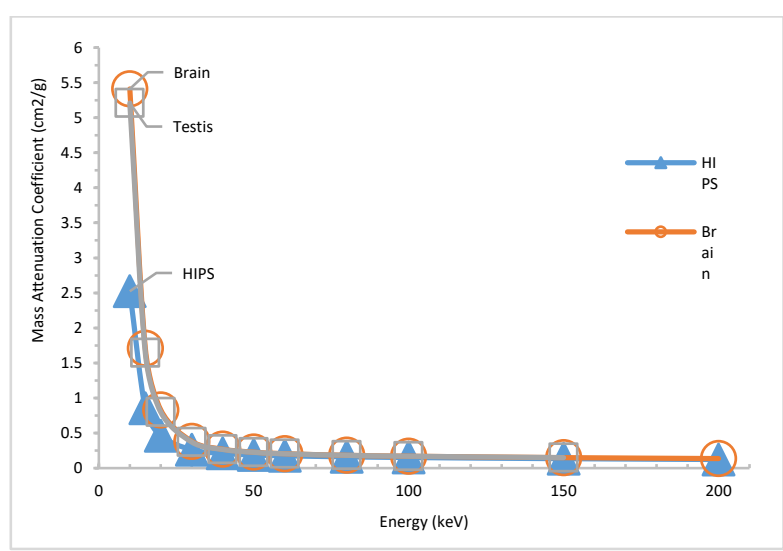

(a) HIPS, brain and testis

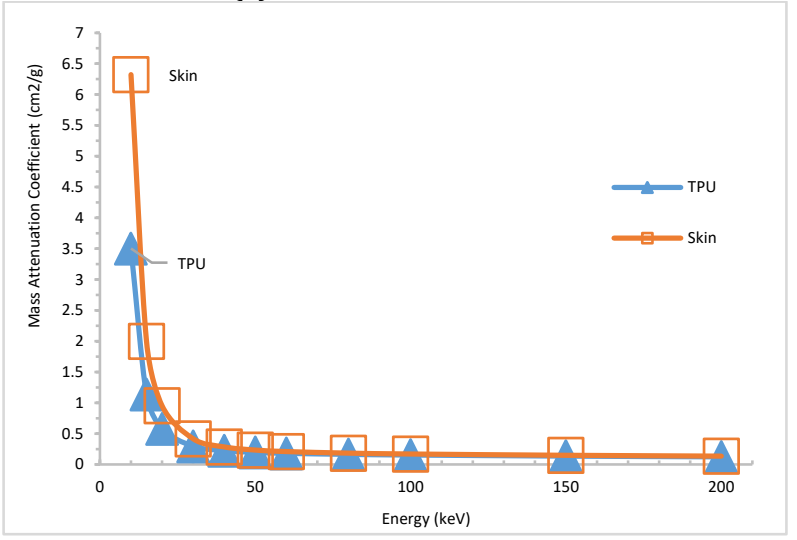

(c) TPU and skin with skin and (d) APS with eye lens, heart, kidney, liver and thyroid. From the graphs, it can be seen that the difference of agreement of the materials with the human tissues in terms of densities cannot considered alone to choose the phantom material.

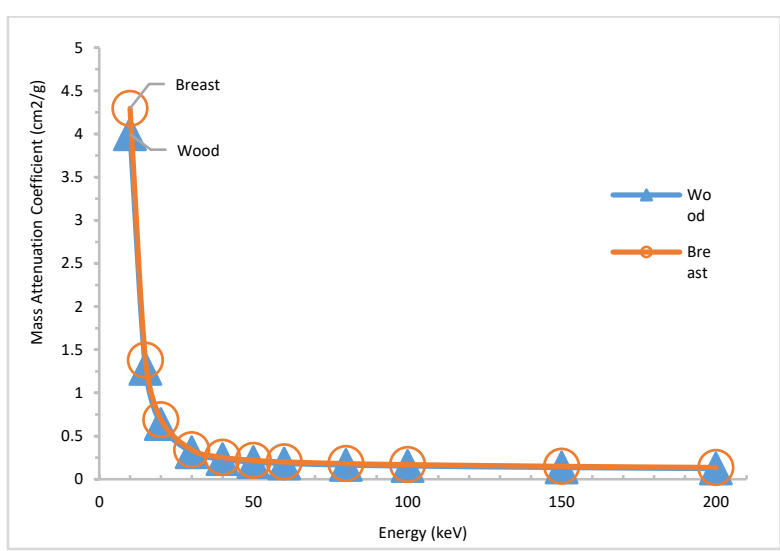

(b) Wood and breast

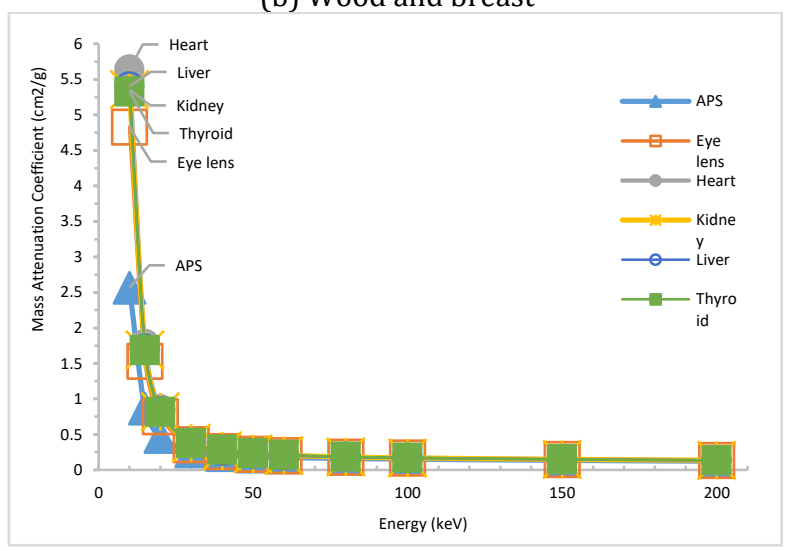

(a) APS, heart, liver, kidney, eye lens and thyroid

Fig. 2: The mass attenuation coefficient of the 3D materials compared with the selected human organs according to the better values of their densities

Furthermore, by ignoring the mass densities of the materials and only taking their attenuation values into account, it can be seen from Fig. 3 that there is a closer match of the mass attenuation coefficients of Polycarbonate (PC) with all human organs under test. The 3D wood material has the most agreement with the Brain, Breast, Testis, Kidney, Thyroid and the TPU material can be used to mimic Eye lens, Heart, Liver and Skin in terms of both the total mass attenuation coefficient and mass 
density. In contrast, the second most agreement material with Brain, Breast, Testis, Kidney and Thyroid was TPU, Wood for Eye lens, Heart, Liver and PA for Skin. The differences between the second and the first agreements are very small; therefore they can also be used as a second choice as a tissue equivalent material for these selected human organs tissue. Fig. 4 and Fig. 5 show the mass attenuation coefficients of the chosen materials (wood and TPU), where the energy extended to cover $10 \mathrm{keV}-200$ $\mathrm{keV}$. Both graphs show a good match between the chosen substitute materials and the selected ICRU's human organs in terms of attenuation properties and mass densities.

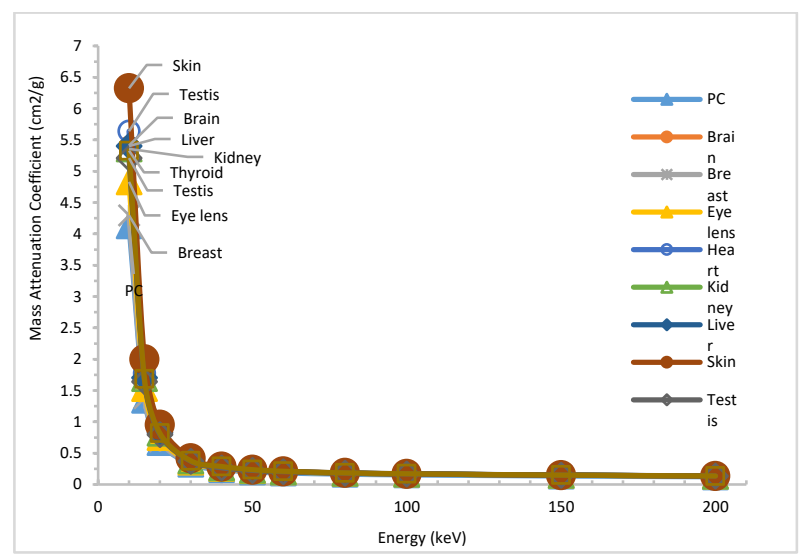

Fig. 3: The mass attenuation coefficients of Polycarbonate (PC) with all human organs under test

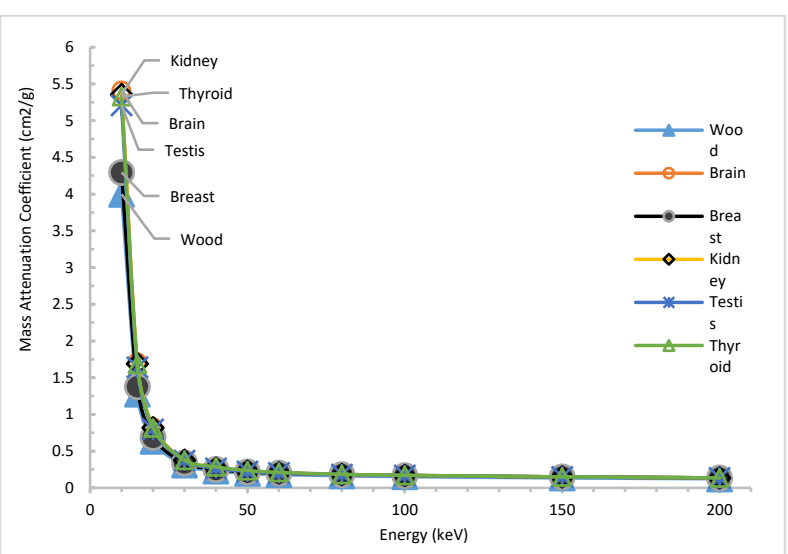

Fig. 4: The mass attenuation coefficient of Wood material compared with the selected human organs

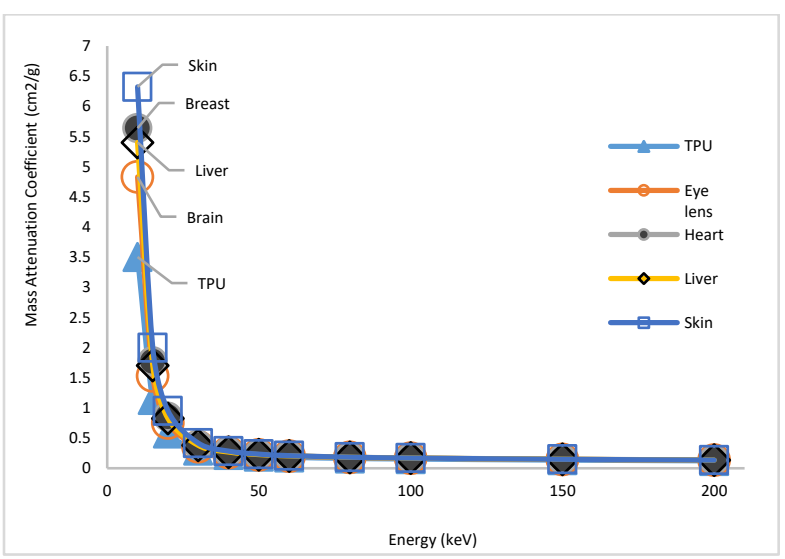

Fig. 5: The mass attenuation coefficient of TPU material compared with the selected human organs

\section{Conclusion}

The 3D Wood printing material is considerably suitable to mimic the human's soft tissues of Breast, Brain, Testis, Kidney, Thyroid and the TPU material is suitable to mimic Eye lens, Heart, Liver and Skin in terms of their elemental compositions, physical density, and the mass attenuation coefficients through calculation and experimental measurement based on this study. Other materials showed good agreements with the human tissues only in terms of the mass attenuation coefficients, but not in the mass density, whereas both of these two quantities are considered when selecting the phantom material (ICRU, 1989).

The advantages of using these materials that all of them are inexpensive and commercially available. In addition, using the 3D printing feature will shorten the time for phantom construction as well as lowering the cost with 10 times less than the usual construction methods and current used materials.

\section{Acknowledgment}

The authors would like to acknowledge financial assistance from the Ministry of Higher Education Malaysia via Fundamental Research Grant Scheme (FRGS: 203.CIPPT.6711386) for supporting this work.

\section{References}

Akça B and Erzeneoğlu SZ (2014). The mass attenuation coefficients, electronic, atomic, and molecular cross sections, effective atomic numbers, and electron densities for compounds of some biomedically important elements at 59.5 $\mathrm{keV}$. Science and Technology of Nuclear Installations, 14(1): 1-8.

Alderson SW, Lanzl LH, Rollins M, and Spira J (1962). An instrumented phantom system for analog computation of treatment plans. The American Journal of Roentgenology, Radium Therapy, and Nuclear Medicine, 87: 185-195.

Alsabbagh M, Ng LY, Tajuddin AA, Manap MA, and Zainon R (2016). Validation of a paediatric thyroid phantom using different multidetector computed tomography models. Journal of Physics: Conference Series, 694(1): 12-47.

Berger M J and Hubbell JH (1999). XCOM: Photon Cross Sections Database. Web Version 1.2. National Institute of Standards and Technology, Gaithersburg, MD 20899, USA. Originally published as NBSIR 87-3597 "XCOM: Photon Cross Sections on a Personal Computer. Available online at: http://physics.nist.gov/xcom

Beutel J, Kundel HL, and Van Metter RL (2000). Handbook of medical imaging: Physics and psychophysics. SPIE Press, Washington, USA.

Borcia C and Mihailescu D (2008). Are water-equivalent materials used in electron beams dosimetry really water equivalent?. Romanian Journal of Physics, 53(7-8): 851-863.

Chanthima N, Prongsamrong P, Kaewkhao J, and Limsuwan P (2012). Simulated radiation attenuation properties of cement containing with BaSO4 and PbO. Procedia Engineering, 32: 976-981.

DeWerd LA and Kissick M (2014). The phantoms of medical and health physics: Devices for research and development. Springer, Berlin, Germany. 
Ferreira CC, Ximenes RE, Garcia CAB, Vieira JW, and Maia AF (2010). Total mass attenuation coefficient evaluation of ten materials commonly used to simulate human tissue. Journal of Physics: Conference Series, 249(1): 12-29.

Gerward L, Guilbert N, Jensen KB, and Levring H (2004). WinXCom - A program for calculating X-ray attenuation coefficients. Radiation Physics and Chemistry, 71(3-4): 653-654.

Hubbell JH (2006). Review and history of photon cross section calculations. Physics in Medicine and Biology, 51(13): R245R262.

Hubbell JH and Seltzer SM (1995). Tables of X-ray mass attenuation coefficients and mass energy-absorption coefficients $1 \mathrm{keV}$ to $20 \mathrm{MeV}$ for elements $\mathrm{Z}=1$ to 92 and 48 additional substances of dosimetric interest. Report No. PB-95-220539/XAB; NISTIR--5632, National Institute of Standards and Technology - PL, Gaithersburg, USA. Available online at: https://www.osti.gov/scitech/biblio/76335

ICRU (1989). Tissue substitutes in radiation dosimetry and measurement. Report 44, International Commission on Radiation Units and Measurements, Maryland, USA.

Jones AK, Hintenlang DE, and Bolch WE (2003). Tissue-equivalent materials for construction of tomographic dosimetry phantoms in pediatric radiology. Medical Physics, 30(8): 2072-2081.

Podgorsak EB (2010). Radiation physics for medical physicists. Springer Science and Business Media, Berlin, Germany.

Shakhreet BZ, Bauk S, Tajuddin AA, and Shukri A (2009). Mass attenuation coefficients of natural Rhizophora spp. wood for $\mathrm{X}$-rays in the $15.77-25.27 \mathrm{keV}$ range. Radiation Protection Dosimetry, 135(1): 47-53.

Singh C, Singh T, Kumar A, and Mudahar GS (2004). Energy and chemical composition dependence of mass attenuation coefficients of building materials. Annals of Nuclear Energy, 31(10): 1199-1205.

Stacey AJ, Bevan AR, and Dickens CW (1961). A new phantom material employing depolymerised natural rubber. The British Journal of Radiology, 34(404): 510-515.

Tousi ET, Bauk S, Hashim, R, Jaafar MS, Abuarra A, Aldroobi KSA, and Al-Jarrah AM (2014). Measurement of mass attenuation coefficients of Eremurus-Rhizophora spp. particleboards for $\mathrm{X}$-ray in the $16.63-25.30 \mathrm{keV}$ energy range. Radiation Physics and Chemistry, 103: 119-125. 